\title{
ARQUEOLOGÍA E HISTORIA. PROPUESTAS PARA UNA REFLEXIÓN CONJUNTA.
}

\author{
Archaeology and History. \\ Proposals for Shared Considerations
}

\author{
Álvaro CARVAJAL CASTRO* \\ Universidad de Salamanca \\ E-mail: carvajalcastro@gmail.com \\ $\mathrm{M}^{\mathrm{a}}$ de los Reyes DE SOTO García** \\ Universidad de Salamanca \\ E-mail: reyesdesoto@usal.es
}

Fecha de recepción: $22-01-2010$

Fecha de aceptación: 05-02-2010

RESUMEN: Tradicionalmente, en la historiografía medieval el estudio de los textos ha relegado la arqueología a un segundo plano. Sin embargo, los arqueólogos medievalistas han realizado en las últimas dos décadas aportaciones teóricas y metodológicas y han producido unos resultados que obligan a replantear abiertamente la relación entre las dos disciplinas, así como las tesis sostenidas hasta ahora por los historiadores. Nuestra comunicación tiene como objetivo exponer los problemas derivados de la ausencia de una base común tanto teórica como metodológica para la Arqueología y la historiografía, así como los problemas a los que nos enfrentamos en nuestras disciplinas, con el objetivo de buscar nexos que permitan la producción de un conocimiento histórico unitario. En este sentido, consideraremos algunas de las cuestiones más candentes del debate sobre la base de nuestras propias investigaciones: el espacio y el poder en el condado de Monzón y el poblamiento rural del valle del Río Almar desde la II Edad del Hierro hasta la Alta Edad Media.

Palabras clave: Arqueología medieval, historiografía, Alta Edad Media, condado de Monzón, valle del Río Almar (Salamanca). 
ABSTRACT: Traditionally, the study of texts has been privileged over archaeology in medieval historiography. However, during the last twenty years, the theoretic and methodological contribution of medieval archaeologists, as well as the results of their research, has lead to a new understanding of the relation between the two disciplines, which also implies the questioning of the old historiographical thesis. This paper will expose the problems that the lack of a common theoretical frame poses in attempt to find the links that could enable us to produce a unitary historical knowledge. We will consider some of the aspects of the debate in the light of our own research work: the study of space and power in the County of Monzón, and the rural settlement of the Valle del Río Almar between the Second Iron Age and the Early Middle Ages.

Keywords: Medieval Archaeology, historiography, Early Middle Ages, County of Monzón, Almar river (Salamanca).

En los últimos años, la historiografía sobre la Alta Edad Media ha estado marcada por la obra de de Chris Wickham Framing the Early Middle Ages ${ }^{1}$. Se trata de una síntesis interpretativa en la que destacan, metodológicamente, el empleo sistemático de la historia comparada y la utilización conjunta de los registros arqueológico y documental. En nuestra comunicación, queremos centrarnos en este último aspecto para señalar, a partir de nuestros trabajos, algunas de las perspectivas, pero también de los problemas, que conlleva la pretensión de aunar ambos registros en el estudio de la época altomedieval. Por un lado, abordaremos la cuestión desde los modelos de poblamiento del Valle del Río Almar, en la provincia de Salamanca; por otro, desde la situación en los territorios comprendidos entre el Cea y el Pisuerga, en la actual provincia de Palencia.

\section{ARQueOlogía E HISTORIA: UNA APROXIMACIÓN AL DEBATE.}

El debate sobre la colaboración entre la arqueología y la historia fue planteando a nivel europeo fundamentalmente a partir de los años 80 del siglo pasado. Fue impulsado gracias al desarrollo metodológico de la arqueología medieval, primero en Francia e Inglaterra en los años 50, y posteriormente, desde mediados de los 60, también en Italia. El interés por el estudio de los despoblados medievales caló primero en Inglaterra, donde en 1952 se fundó el Deserted Medieval Villages Research Group-luego simplemente Medieval Villages Research Group. Unos años después, en Francia, tendría lugar la creación del $C R A M^{2}$. En Italia, el peso de la arqueología clásica marcaba la agenda de la investigación, pero la labor de arqueólogos como G. Bognetti o N. Lamboglia, así como la influencia de la British School at Rome o la École Française de Rome sirvió para potenciar la disciplina ${ }^{3}$. 
Este interés se tradujo pronto en la creación de ámbitos de publicación y de discusión propios. Cabe mencionar, por ejemplo, las diversas revistas sobre Arqueología Medieval que nacieron al calor de los primeros esfuerzos como Medieval Archaeology (1957), Archéologie Médievale (1971) o Archaeologia Medievale (1974). Las iniciativas editoriales y los foros de debate se han multiplicado desde entonces. En este sentido, una de las principales preocupaciones hoy en día es la de dar a conocer los resultados de las excavaciones de gestión, cuyo aportación al conocimiento histórico ha sido poco aprovechada a pesar de ser cuantitativa y cualitativamente importante 4 .

En 1986 Paolo Delogu daba por concluido el periodo fundacional de la arqueología medieval italiana y, si bien reconocía que los resultados no estaban a la altura de las primeras expectativas, apuntaba ya a la posibilidad de construir un discurso histórico propio desde las fuentes arqueológicas. El resultado se plasmaría unos años después en la publicación La storia dell'alto Medioevo italiano alla luce della archeologia, en la que el propio Delogu señalaba cómo habían contribuido los avances en los estudios sobre la cerámica, sobre la arqueología urbana, etc ${ }^{5}$.

En aquel mismo artículo, Paolo Delogu dejaba constancia de una preocupación entonces ya presente: la relación entre la interpretación histórica y la interpretación arqueológica ${ }^{6}$. Señalaba como el desarrollo de las investigaciones había forzado a un arqueólogo como Francovich, inicialmente conciliador, a tomar una posición más marcada en relación con la necesidad de la arqueología de elaborar un discurso histórico propio y esperar que las condiciones de desarrollo de cada disciplina permitieran una aproximación conjunta. En los años 80 se experimentaron diversas líneas en este sentido. Algunos autores, como Hodges, abogaron en un principio por una relación más estrecha entre la Arqueología y la Antropología ${ }^{7}$ En otros casos, como el español, Miquel Barceló señalaba la necesidad de un desarrollo independiente de las imposiciones de las tesis historiográficas como requisito indispensable para la construcción de la disciplina ${ }^{8}$. Francovich y Hodges lo formularon como la necesidad de encontrar un ámbito de estudio que resultara igualmente atractivo para historiadores y arqueólogos?. Lo cierto es que, precisamente, esta publicación se insertaba en una línea de investigación que se probaría muy prolífica en este sentido: el estudio del incastellamento.

Las tesis de Pierre Toubert sobre el incastellamento en el norte de Italia manifestaban el interés que se tenía desde la historia por el estudio del espacio. Un ámbito en el que lo desarrollos propios de la arqueología, bajo las formas de la Arqueología Espacial y, posteriormente, la Arqueología del Paisaje, hicieron posible una temprana convergencia de intereses ${ }^{10}$. En 1980, Fossier y Chapelot publicaban Le village et la maison, una obra en la que los autores abogaban por la colaboración interdisciplinar como vía de acceso a un conocimiento más preciso de la realidad aldeana medieval. El problema, 
tanto de las propuestas de Toubert como de las de Fossier, es que basaban sus teorías en definiciones elaboradas desde los textos, y éstas no se correspondían con la realidad material que proporcionaban las excavaciones arqueológicas. Así lo señalaría Zadora-Rio en 1995, en un artículo en el que comparaba las evidencias documentales y materiales que se manejaban en la definición de las aldeas medievales ${ }^{11}$. En este sentido, el riesgo que se corría era el de duplicar términos y conceptos que, a pesar de las diferencias que mostraban en el registro, eran fruto de una única realidad histórica. Igualmente, se corría el riesgo de malinterpretar los procesos de transformación del poblamiento. Las aportaciones de Francovich y Hodges constituían precisamente una crítica a las cronologías y a los procesos propuestos por Toubert.

En los años 90, el auge de las teorías contextuales permitió una nueva aproximación al problema. Las propuestas de Moreland, sintetizadas en su obra de 2001, apuntaban hacia la necesidad de una equiparación epistemológica de los dos registros, es decir, de considerarlos en un mismo nivel en el acceso al conocimiento histórico, como base para la interpretación conjunta ${ }^{12}$. Entre sus propuestas, y a partir de una deconstrucción del predominio del Texto sobre el Objeto, figuraba una reconsideración del valor de lo escrito en su contexto.

En términos generales, la arqueología ha conocido un dinamismo teórico que ha contribuido en gran medida a la interpretación histórica. Hoy abordamos de manera más rica temas tales como la formación de las aldeas, el ejercicio y la simbolización del poder, la noción de comunidad y de identidad, la transformación de las redes de poblamiento o la construcción de entidades territoriales de carácter político ${ }^{13}$. Las aplicaciones que se han hecho, por ejemplo, de la teoría de sistemas o del funcionamiento de las relaciones centro-periferia constituyen campos provocadores para la reflexión histórica ${ }^{14}$.

En el caso español en concreto, la vigencia de las teorías de Sánchez Albornoz sobre la despoblación del valle del Duero o la necesidad de reforzar las tesis contrarias habían limitado el papel de la arqueología en el debate ${ }^{15}$. A pesar de las aportaciones que entonces hicieron algunos arqueólogos ${ }^{16}$, las excavaciones han jugado y están jugando un papel mucho más importante a la hora de matizar las alternativas a la tesis de la despoblación y a la formulación de nuevos modelos ${ }^{17}$. Los modelos basados en la colonización y la idea de frontera, o la gran línea de investigación desarrollada por García de Cortázar y su escuela bajo la noción genérica de "organización social del espacio", a pesar de sus importantes aportaciones, constituyeron ya un objeto de crítica de tesis doctorales que, al tradicional interés por la configuración del espacio, aunaban una sensibilidad mayor hacia la arqueología $a^{18}$. Tanto es así que recientemente se ha llegado a proponer un "giro arqueológico" en la historiografía española 19 . 
En definitiva, a lo largo, sobre todo, de estos últimos veinte años, se ha constituido en nuestro país una expectativa de conocimiento que, aún consciente de las dificultades, busca la forma de integrar en modelos interpretativos comunes las aportaciones del registro arqueológico y de la documentación escrita. Sin embargo, el mero voluntarismo no es suficiente para superar los obstáculos que plantea la práctica de estas disciplinas. Sólo al confrontarlo con las evidencias específicas podemos valorar el verdadero alcance de la utilización conjunta de arqueología e historia.

\section{LOS CASOS DE ESTUDIO}

A pesar de las diferencias entre ambos espacios, las zonas que hemos propuesto para nuestros trabajos comparten una serie de rasgos destacables. Sobre todo, que a pesar de la existencia de intervenciones arqueológicas previas, el nivel de información del que disponemos no es muy alto. Puesto que las perspectivas de estudio son distintas, esto nos afecta en diversa medida. Sin embargo, algunos de los rasgos que subyacen detrás de estas carencias son comunes. Para empezar, por ejemplo, los problemas de visibilidad afectan a ambas zonas, tanto a nivel técnico (prospectivo), como en lo que se refiere a la visibilidad de los grupos sociales a través de los vestigios materiales. A nivel autonómico, la Comunidad de Castilla y León no ha conocido un desarrollo urbanístico o vial similar, en términos relativos, al de otras regiones. Esto supone que no han sido tantas las intervenciones de gestión realizadas, las cuales, sin embargo, no dejan de constituir una parte importante de las aportaciones recientes en cuanto a la cantidad y variedad del registro disponible. Igualmente, falta todavía por profundizar en el estudio crono-tipológico de las cerámicas altomedievales. De todos modos, es necesario matizar lo que ocurre en cada una las zonas.

\section{LOS TERrITORIOS DE LOS CONDADOS DE MONZÓN Y SALDAÑA CARRIÓN}

El territorio entre el Cea y el Pisuerga conoció, a lo largo de los siglos X y XI, el establecimiento de dos grandes condados, el de Monzón y el de Saldaña-Carrión ${ }^{20}$. También el de distintos monasterios desde el siglo X-San Isidro de Dueñas, Santa Eufemia de Cozuelos, San Román de Entrepeñas, entre otros - que nos permiten hoy contar con un cierto registro documental para la región ${ }^{21}$. Sin embargo, ni el ámbito temporal que abarca la documentación ni la densidad de la misma nos permiten una lectura retrospectiva sencilla mínimamente fiable. El trabajo de reconstrucción de la red de poblamiento y de la posible organización del territorio se basa en la interpretación de términos y expresiones como alfoz, territorio, término, suburbio, o en la reconstrucción de 
flujos económicos, fiscales, etc ${ }^{22}$. Su presencia, relativamente abundante en la documentación, nos permite establecer siquiera una mínima diferencia significativa para definir las relaciones entre distintos núcleos. Una aproximación en este sentido requiere de un estudio de la percepción del espacio a través de las referencias toponímicas o geográficas empleadas. Esto, a su vez, nos ayuda a definir los ámbitos de actuación, tanto de las comunidades como de las aristocracias locales y a profundizar en el conocimiento de la construcción de la identidad: cómo una comunidad puede ser definida en base a su relación con un espacio. Los problemas de visibilidad de ambos grupos sociales, más allá de los representantes de las aristocracias más destacadas, suponen también un reto: el de la construcción interpretativa de una diferencia no evidente en los documentos. Lo interesante sería llegar más allá de la cuantificación del valor económico de las transacciones y encontrar el significado de determinados intercambios, como las menciones a objetos entregados en concepto de robra en donaciones y compraventas.

En contadas ocasiones, documentos excepcionales (para esta época) nos permiten también estudiar más detenidamente los comportamientos de determinados individuos o colectivos. En último término, el objetivo consiste en definir las fórmulas de implantación del poder sobre el espacio a través de las distintas escalas de ejercicio del mismo (local, supralocal, regional). De esta forma, pretendemos abordar la dinámica interna de las entidades políticas que se articulan sobre el territorio y estudiar cual es el grado de implantación y de efectividad de los poderes condales.

La aportación de la arqueología sería muy importante, puesto que nos permitiría esclarecer los procesos de creación de las redes de poblamiento vigentes a finales del siglo IX, época del primer desarrollo de estos condados. Sin embargo, y a pesar de una relativa abundancia de intervenciones (normalmente prospecciones o catas prospectivas), las posibilidades de estudio son limitadas. Existen una serie de yacimientos excepcionales que han concentrado el interés de los investigadores y de las administraciones, pero que ha desfigurado el territorio que poco se corresponde con la realidad de los hallazgos. La presencia de grandes villas monumentales relativamente bien conservadas, como La Olmeda (Pedrosa de la Vega), ha sobredimensionado la importancia de los restos romanos, proyectando una falsa imagen sobre el resto de la provincia ${ }^{23}$. El abuso del término villa ha influido en la interpretación de numerosos yacimientos, contribuyendo a ocultar otros tipos de hábitats de época romana y visigoda. Igualmente, para la época altomedieval, gran parte de las publicaciones se han visto condicionadas por las tesis despoblacionistas. La datación de materiales en base a las noticias de repoblación como término post quem condicionaron su estudio e impidieron reconocer las cerámicas de los siglos VIII y IX. Esto supone que apenas hay en la bibliografía yacimientos adscritos ese periodo ${ }^{24}$. El interés por la presencia vi- 
sigoda en la región nos permite al menos contar con más información para esa época ${ }^{25}$.

Uno de los casos más significativos es el de El Castellar, en Villajimena ${ }^{26}$. Se trata de un poblamiento en lo alto de un pequeño cerro, en nada comparable a la ubicación de otros núcleos como Monte Cildá o Monte Bernorio, de los que ahora hablaremos. Las excavaciones revelaron los restos de dos necrópolis, una atribuida a época visigoda, otra a los siglos IX y X, así como restos de estructuras constructivas. No se pudo establecer la estratigrafía y resulta complicado interpretar la secuencia del poblamiento, si bien los indicios apuntan hacia una continuidad de ocupación.

En el norte de la provincia existen núcleos de habitación en altura de características muy diferentes. Monte Cildá o Monte Bernorio representan, en términos de paisaje, referencias de relativa importancia, constatada desde luego para el periodo visigodo ${ }^{27}$. Se trata de un entorno en el que se conoce la presencia de otros castros como el de Gama ${ }^{28}$. Las excavaciones llevadas a cabo en los dos primeros se centraron en la excavación de lo que parecían ser las murallas, por lo que falta un estudio extensivo que nos permita comprender mejor la naturaleza de su ocupación. Además sería necesario establecer la relación entre estos núcleos y su entorno, y la de los núcleos entre sí, puesto que se han estudiado de manera independiente y sólo se han integrado a nivel interpretativo en el discurso sobre la presencia cántabra y las campañas militares visigodas.

A nivel regional, se han realizado algunas prospecciones en espacios más amplios. Por ejemplo, las realizadas en la ribera izquierda del Carrión entre La Serna y Saldaña documentaron la presencia de yacimientos de época romana, tardoantigua y altomedieval ${ }^{29}$. Harían falta estudios, siquiera de prospección intensiva, que nos permitieran definir mejor el tipo de poblamiento de esta zona, para la que no hay documentación en los siglos X y XI.

Constatar y definir la existencia de estos procesos constituiría un paso muy importante en el estudio de la región, puesto que pondría en relieve las dinámicas de producción social del espacio y, a través de éstas, el grado de integración de los distintos territorios, la densidad y la complejidad de las redes de ejercicio del poder. Con ello, podríamos incluso establecer un término para la comparación entre las pretensiones de un poder, al que se le supone un cierto grado de autonomía, y la realidad social existente; es decir, para valorar la adecuación del poder en los distintos niveles en los que despliega su actuación y en función de las distintas fórmulas que adopta para su ejercicio. Sin embargo, los tiempos de la investigación arqueológica no nos permiten abordarla de manera intensiva en un estudio que, en términos espaciales, pretende abarcar un ámbito tan amplio como el que la documentación, también debido a sus características, no sólo permite, sino que requiere. 


\section{El valle del Río Almar (Salamanca)}

El trabajo que estamos llevando a cabo en el valle del Río Almar se centra en el poblamiento prerromano, romano y altomedieval. La investigación arqueológica no ha tenido muy presente las zonas cercanas a Salamanca, por lo que esta zona carece de estudios específicos. Hay que señalar que la aportación de las intervenciones de urgencia es relativamente importante para nuestro trabajo como punto de partida. Se han realizado prospecciones y excavaciones en los términos municipales de Alconada, Coca de Alba, Garcihernández, Peñarandilla y Ventosa del Río Almar, lo que nos permite partir, a través de la consulta de los informes, con una idea aproximativa del área de estudio. Se han conocido así yacimientos como la Vega de Santa Bárbara (Alconada), una villa de la que se hallaron las instalaciones termales en el sondeo, o la necrópolis de La Serna (Matamala, Garcihernandez) ${ }^{30}$. Finalmente, a diferencia de los territorios palentinos, se trata de una zona que carece de documentación escrita para la época, por lo que los estudios se basan por completo en la labor arqueológica.

El proyecto se sustenta en las prospecciones arqueológicas de todos los términos municipales que abarca el río Almar a su paso por la provincia de Salamanca. Contamos para el caso salmantino con algunos ejemplos de prospecciones de tipo intensivo y extensivo ${ }^{31}$. En base a los mismos, pretendemos comprobar si los patrones de asentamiento establecidos en la comarca de La Armuña (Salamanca) se repiten en otras zonas de la provincia. El hecho diferenciador con respecto al trabajo citado es la existencia de un castro prerromano, lo que puede ser interesante al ser una nueva variable de estudio en la medida en que pueda haber supuesto un cambio de patrones de hábitat.

Las primeras prospecciones realizadas sobre el terreno nos han permitido formular algunas ideas de partida. En primer lugar, se aprecia la existencia de hábitats y materiales de distinto tipo que permiten constatar una ocupación del territorio dilatada en el tiempo. El siguiente paso, a partir de los datos recopilados a pie de campo y a través de los análisis de los restos recuperados, consiste en desentrañar los modelos habitacionales para diferenciar los distintos tipos de ocupación, tanto en un sentido diacrónico como sincrónico. Las dificultades fundamentales estriban en el estudio de las cerámicas. Las producciones más abundantes que encontramos en los yacimientos son las cerámicas comunes de cocina, difíciles de datar mediante criterios macroscópicos debido a que presentan características muy similares a lo largo del tiempo. Es difícil, por lo tanto, discernir la cronología o su tipología a partir de estos restos. Aunque se han realizado algunos estudios, pensamos que sería necesario profundizar en este aspecto ${ }^{32}$. 
En muchos casos dependemos, pues, de que exista o no la posibilidad de recuperar otro tipo restos. Por ejemplo, algunas informaciones orales recogen el hallazgo, durante la realización de pozos o al arar las tierras, de varias estelas, una de las cuales se ha conservado ${ }^{33}$. En relación con el resto del material obtenido, suponen una aportación cualitativa importante. Las prospecciones realizadas en el castro de la Cuesta de Santa Ana encontraron una fíbula de caballito que, aunque sin duda singular en el contexto, fue hallado en superficie y, por tanto, descontextualizado, lo que limita su aportación ${ }^{34}$. Se encontraron, igualmente descontextualizadas, dos pizarras visigodas provenientes de San Vicente del Río Almar ${ }^{35}$.

Para finalizar, hay que destacar los hallazgos en el término de Garcihernández. En dicho término se encuentra la Cuesta de Santa Ana, un castro cuya primera ocupación se documenta en la Segunda Edad del Hierro y que

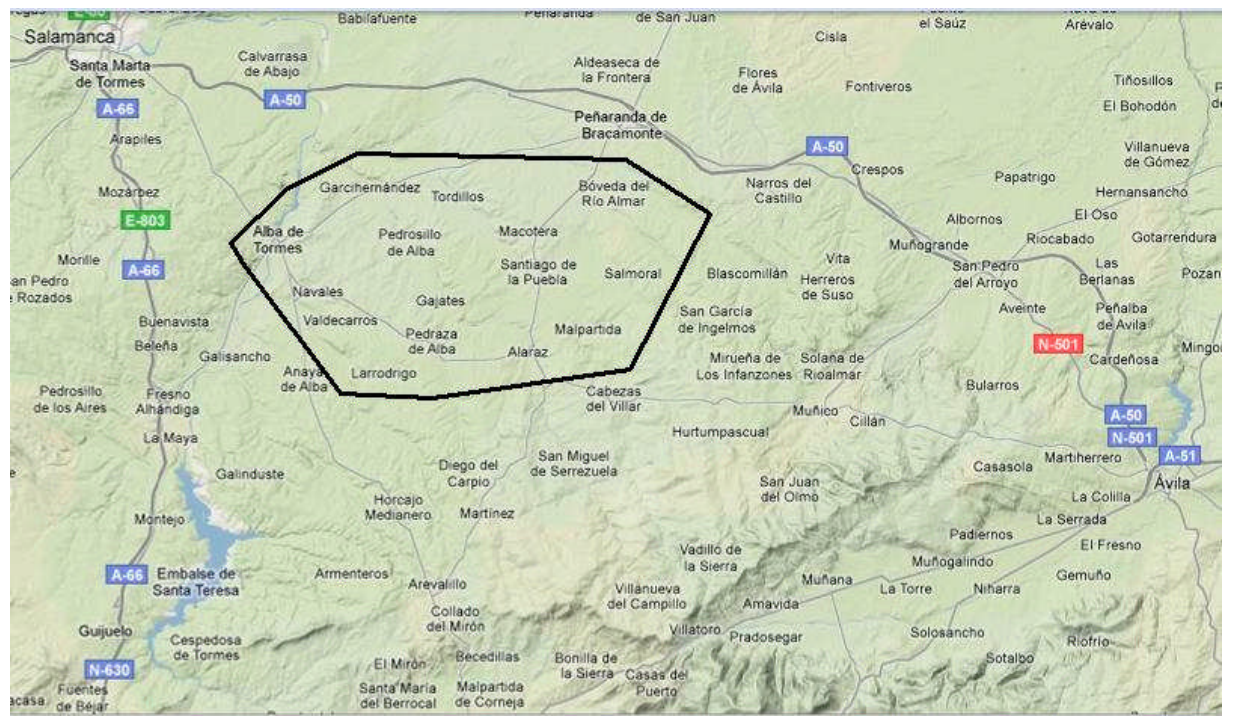

Fig. 1. El valle del río Almar en la provincia de Salamanca.

parece experimentar una reocupación altomedieval ${ }^{36}$. Entre ambas épocas, el poblamiento romano parece centrarse en el llano, próximo castro. Las obras de mejora del regadío permitieron descubrir restos de lo que se supone una villa romana. Los restos nos plantean dos problemas. En primer lugar, definir adecuadamente la secuencia y la naturaleza de la ocupación en cada 
momento. ¿De qué forma incidió la instalación de una villa romana en la configuración del territorio? ¿Se mantuvo la ocupación, siquiera superficial, del castro? ¿Qué naturaleza habría tenido de ser así? Por otro lado, los restos de una ermita resultan igualmente problemáticos. ¿Podemos datarla en época medieval o son restos de una construcción de época posterior? En cualquier caso, evidencian la importancia del lugar como referencia espacial.

\section{PROBLEMAS Y PERSPECTIVAS COMUNES}

Existen, pues, una serie de perspectivas comunes para las que se plantean preguntas similares, matizadas en función del contexto, pero con las mismas preocupaciones de fondo. Por un lado, la necesidad de identificar elementos que nos permitan visibilizar restos relevantes ${ }^{37}$. Por otro, lo que Delogu expresaba como la necesidad de identificar las funciones de la vida social que convergen en la producción de un objeto, haciendo esto extensivo a la producción de un texto ${ }^{38}$. Como productos sociales, todo texto y todo objeto puede, potencialmente, reflejar en positivo -por sus características propias y sus funciones- o en negativo -por las relaciones sociales en que se inserta-, los condicionantes sociales del momento de su producción. Sin embargo, no todas ellas se expresan en el registro, primero porque su materialización sólo nos permita conocer parcialmente su funcionalidad, y segundo porque los contextos materiales o textuales en los que es hallado no nos permiten reconstruir más que parcialmente las interrelaciones. Y todo ello sin perder de vista las transformaciones -funcionales o simbólicas- que los elementos pudieron experimentar con el paso del tiempo.

En este sentido, es posible hablar de una complementariedad entre el registro arqueológico y el registro documental, en la medida en que uno u otro puedan aportar informaciones exclusivas. Pero debemos también considerar de qué forma se solapan las posibilidades de interpretación de uno y otro. Es desde esta base que debe partir la consideración de una equiparación epistemológica que nos permita un uso conjunto de los registros. No sólo a partir de las preguntas que se puedan formular en aquellos casos en los que se solapan los registros, sino a partir de planteamientos heurísticos que permitan situar los dos tipos de informaciones -exclusivas e comunes- en un plano interpretativo. 
Esas preguntas responden a preocupaciones comunes. Por ejemplo, nos interesan determinadas secuencias ocupacionales en la medida en que nos permiten revelar transformaciones sociales locales y ligarlas con procesos más amplios. La introducción de fórmulas de organización social ajenas a las condiciones preexistentes podría informarnos sobre los procesos de adecuación o resistencia a las mismas, si bien es cierto que por los restos materiales de que disponemos resulta muy complicado y apenas hay rastros en la documentación que manejamos. Un ejemplo lo constituyen las villae como la de La Serna (Salamanca). Pero la pregunta se refleja de igual modo a la hora de considerar el grado de implantación de los poderes condales sobre el territorio, e incluso sería un término para la comparación entre el de Monzón y el de Saldaña-Carrión, que parecen responder a necesidades de poder diferentes.

Estos planteamientos son posibles gracias a modelizaciones previas que es necesario contrastar con los contextos concretos que manejamos, como así reflejan las pretensiones del trabajo sobre el Río Almar. Este tipo de análisis espaciales a nivel regional constituiría, por otra parte, una gran aportación a los territorios palentinos, ya que permitiría una síntesis arqueológica más comprensiva que la que se basa en yacimientos singulares. De ahí la necesidad de potenciar en ambos casos las investigaciones arqueológicas.

\section{NOTAS}

* Esta comunicación ha sido realizada en el marco de una Beca de Formación de Profesorado Universitario gestionada por el Ministerio de Educación.

** Beneficiaria de la Beca de Formación de Personal Investigador de la Junta de Castilla y León, Universidad de Salamanca.

${ }^{1}$ WICKHAM, C., Framing the Early Middle Ages, Oxford, 2005.

${ }^{2}$ QUIRÓS, J.A. y BENGOETXEA, B., Arqueología III. Arqueología Post-Clásica, Madrid, 2006.

${ }^{3}$ DELOGU, P., "La fine del mondo antico e l'inizio del medioevo: nuovi date per un vecchio problema", en La storia dell'alto medioevo italiano (VI-X secolo) alla luce dell'archeologia. Convegno Internazionale (Siena, 2-6 dicembre, 1992), Firenze, 1994, pp. 7-23.

${ }^{4}$ A este problema responden iniciativas como la creación de la serie "Documentos de Arqueología" como parte del proyecto de investigación "Arqueología de las aldeas". Su primer número, que recoge las ponencias y comunicaciones al congreso Arqueología de las aldeas en 
la Alta Edad Media, celebrado en Vitoria en noviembre de 2008, ha sido recientemente presentado. Se puede obtener más información sobre el proyecto en la página web www.arqueologiadelasaldeas.com [Fecha de consulta: 19-01-2010]. Véase también QUIRÓS, J., The Archaeology of Villages in Early Medieval Ages, Bilbao, 2009.

${ }^{5}$ DELOGU, P., "La fine del mondo antico...".

${ }^{6}$ DELOGU, P., “Archeologia medievale: un bilancio di vent'anni”, Archeologia Medievale, XIII, 1986, pp. 493-505.

${ }^{7}$ HODGES, R., "Method and Theory in Medieval Archaeology", Archeologia Medievale, IX, 1982.

${ }^{8}$ BARCELÓ, M., Arqueología medieval en las afueras del "medievalismo", Barcelona, 1988.

${ }^{9}$ FRANCOVICH, R. y HODGES, R., "Acheologia e storia del villaggio fortificato di Montarrenti (SI): un caso o un modello?”, Archeologia Medievale, XVI, 1989, pp. 15-38.

${ }^{10}$ HODDER y ORTON, Spatial Analysis in Archaeology, Cambridge, 1976; CLARKE, D. L., Spatial Archaeology, Londres, 1977; TILLEY, C., A phenomenology of landscape: places, paths, and monuments, Oxford, 1994.

${ }^{11}$ ZADORA-RIO, E. (1995), "Le village des historiens et le village des archéologues", en MORNET, E. (ed.), Campagnes médiévales: l'homme et son espace. Études offertes à Robert Fossier, Paris, pp. 145-154.

${ }^{12}$ MORELAND, J., Archaeology and Text, Londres, 2001.

${ }^{13}$ CHAVARRÍA, A., El final de las villae en Hispania (siglos IV-VIII), Brepols, Turnhout, 2007; CHRISTIE, N., Landscapes of Change. Rural Evolutions in Late Antiquity and the Early Middle Ages, Podstow, 2004; FERNÁNDEZ MIER, M., Génesis del territorio en la Edad Media: arqueología del paisaje y evolución histórica en la montaña asturiana, Oviedo, 1999; FRANCOVICH, R. y HODGES, R., Villa to village: the transformation of the Roman countryside in Italy, c. 400-1000, Londres, 2003; GARCÍA CAMINO, I., Arqueologia y poblamiento en Birkaia, siglos VI-XII. La configuración de la sociedad feudal, Bilbao, 2002; GLEDHILL y BENDER, State and Society. The Emergence and Development of Social Hierarchy and Political Centralization, Londres, 1988; LÓPEZ QUIROGA, El final de la Antigüedad en la Gallaecia: la transformación de las estructuras de poblamiento entre Miño y Duero (Siglos V al X), La Coruña, 2004; QUIRÓS, "La génesis del paisaje medieval en Álava: la formación de la red aldeana”, Arqueología y Territorio Medieval, 13 (1), 2006, pp. 49-89; VIGIL-ESCALERA, A., "Aspectos sobre la cultura material en Hispania (ss. V-VIII). Problemas de visibilidad/invisibilidad en el registro arqueológico", en LÓPEZ QUIROGA, J.; MARTÍNEZ TEJERA, A. M., MORÍN DE PABLOS, J. (eds.), Gallia e Hispania en el contexto de la presencia germánica (ss. V-VIII). Balance y perspectivas, Oxford, 2006, p.89-108.; VIGILESCALERA, A., "Granjas y aldeas tardoantiguas y altomedievales de la Meseta. Configuración espacial, socioeconómica y política de un territorio rural al norte de Toledo (ss. V-X d.C.), Archivo Español de Arqueología, 80, 2007, pp. 239-285.

${ }^{14}$ KRISTIANSEN y ROWLANDS, Social Transformation in Archaeology. Global and Local Perspectives, Londres, 1998 
${ }^{15}$ SÁNCHEZ ALBORNOZ, C., Despoblación y repoblación del valle del Duero, Buenos Aires, 1966.

${ }^{16}$ REYES TÉLLEZ, y MENÉNDEZ ROBLES, M.L., "Aspectos ideológicos en el problema de la despoblación del valle del Duero”, en ARCE, J. y OLMOS, R. (eds.), Historiogafía de la arqueología y de la Historia Antigua en España (siglos XVIII-XX), Madrid, 1991, pp. 199207.

${ }^{17}$ FERNANDEZ MIER, M., Génesis del territorio en la Edad Media: arqueología del paisaje y evolución histórica en la montaña asturiana, Oviedo, 2000; GARCÍA CAMINO, I., Arqueología y poblamiento en Birkeaia, siglos VI-XII. La configuración de la sociedad feudal, Bilbao, 2002.

${ }^{18}$ MARTÍN VISO, I., Poblamiento y estructuras sociales en el norte de la Península Ibérica: (siglos VIXIII), Salamanca, 2000; ESCALONA, J., Sociedad y territorio en la Alta Edad Media Castellana: la Formación del Alfoz de Lara, Oxford, 2002.

${ }^{19}$ ESCALONA, J., “The early Castilian peasantry: an archaeological turn?”, Journal of Medieval Iberian Studies, 1:2, 2009, pp. 119-145.

${ }^{20}$ Una aproximación en MARTÍNEZ DÍEZ, G., "Los condados altomedievales: Castilla, Monzón y Carrión", en Repoblación y Reconquista. Actas del III Curso de Cultura Medieval. Aguilar de Campoo, septiembre de 1991, Madrid, 1993, pp. 115-125. En cualquier caso, se trata de una aproximación desde la historia política.

${ }^{21}$ PÉREZ CELADA, J.A., Documentación del monasterio de San Zoilo de Carrión, Palencia, 19861987, 2v.; REGLERO DE LA FUENTE, C.M., El Monasterio de San Isidro de Dueñas en la Edad Media: un priorato cluniacense hispano (911-1478). Estudio y colección documental, León, 2005; RUIZ ASENCIO, J.M.; RUIZ ALBI, I. y HERRERO JIMÉNEZ, M., Colección documental del Monasterio de San Román de Entrepeñas (940-1608), León, 2005.

22 ESCALONA, J., "Unidades territoriales supralocales: una propuesta sobre los orígenes del señorío de behetría “, en ESTEPA DÍEZ, C. y JULAR PÉREZ-ALFARO, C., Los señoríos de behetría, Madrid, 2001, pp. 21-46; ESTEPA DÍEZ, C., "La behetría y el poder regio", en Idem, pp. 47-72.

${ }^{23}$ PALOL, P., La villa romana de La Olmeda de Pedrosa de la Vega (Palencia): guía de de las excavaciones, Palencia, 1982. Ver también ABÁSOLO ÁLVAREZ, J.A., La necrópolis Norte de La Olmeda (Pedrosa de la Vega, (Palencia), Palencia, 2004.

${ }^{24}$ Por ejemplo, LAMALFA DÍAZ, C.; PEÑIL MÍNGUEZ, J. (1995), “Testar medieval "Casa del Conde", Olleros de Paredes Rubias, Palencia", en Actas del III Congreso de Historia de Palencia. T. I, Prehistoria, arqueología e historia antigua, Palencia, pp. 563-578.

${ }^{25}$ Una visión general, con una relación de restos en ALONSO ÁVILA, A., "En torno a la visigotización de la provincia palentina”, PITTM, 53, 1985, pp. 267-295.

${ }^{26}$ GARCÍA GUINEA, M.A., et al., "El Castellar, Villajimena (Palencia): Memoria de excavaciones", PITTM 23,1963 , pp. 123-158.

${ }^{27}$ GARCÍA GUINEA, M.A.; et al., "Excavaciones en Monte Cildá, Olleros de Pisuerga (Pa- 
lencia)", PITTM, 26, 1968, pp. 1-68; GARCÍA GUINEA, M.A., IGLESIAS GIL, J.M. y CALOCA, P., 1973, "Excavaciones de Monte Cildá, Olleros de Pisuerga (Palencia): Campaña de 1966 a 1969”, PITTM, 34, pp. 1-95; SAN VALERO APARISI, J., "Monte Bernorio. Aguilar de Campoo (Palencia): Campaña de estudio en 1959”, PITTM, 24, 1965, pp. 97.135

${ }^{28}$ ALONSO CRESPO, G. et al., El Castro de los Barahones, Gama (Palencia), Sautuola: Revista del Instituto de Prebistoria y Arqueología Sautuola, 5, 1986-1988, pp. 71.80

${ }^{29}$ CORTÉS, J., RÍOS, D., “Aportación a la carta arqueológica de Palencia: Yacimientos en la margen izquierda del Río Carrión, entre Saldaña y La Serna”, PITTM, 43, 1979, pp. 41 60 .

${ }^{30}$ STRATO, Gabinete de Estudios sobre Patrimonio Histórico y Arqueológico. Prospección arqueológica intensiva de la red de riego para la mejora y modernización del regadío de la comunidad de regantes "Vegas del Almar". Términos municipales de Alconada, Coca de Alba, Garcihernández, Peñarandilla y Ventosa del Río Almar (Salamanca). Salamanca, 2003, Informe inédito depositado en el Museo Provincial; STRATO, Gabinete de Estudios sobre Patrimonio Histórico y Arqueológico. Informe técnico. Trabajos de excavación arqueológica integrados en el proyecto de la Red de Riego para la mejora y modernización del regadio de la comunidad de regantes "Vegas del Almar". Términos municipales de Alconada, Coca de Alba, Garcihernández, Peñarandilla y Ventosa del Río Almar (Salamanca). Salamanca, 2003, Informe inédito depositado en el Museo Provincial

${ }^{31}$ ARIÑO, E.; RIERA, S.; RODRÍGUEZ, J., "De Roma al Medievo. Estructuras de hábitat y evolución del paisaje vegetal en el territorio de Salamanca", Zephyrus, 55, 2002, pp. 283309; ARIÑO, E., Ager Salmanticensis: prospecciones arqueológicas en el entorno de la ciudad de Salamanca. Años 1993-1996, Salamanca, 2002, Informe inédito depositado en el Museo Provincial de Salamanca; BARBERO, L.; Poblamiento de época romana y visigoda en el valle del Alagón (Salamanca): análisis territorial. Trabajo de Grado inédito, Salamanca, 2004; ARIÑO, E., "Modelos de poblamiento rural en la provincia de Salamanca entre la Antigüedad y la Alta Edad Media”, Zephyrus, 59, 2006, pp. 317-337. ARIÑO, E.; HERNÁNDEZ, J., "El poblamiento romano y visigodo en el territorio de Salamanca. Datos de una prospección intensiva", Zephyrus, 50, 1997, pp. 225-245.

${ }^{32}$ LARRÉN, H. et al., "Ensayo de sistematización de la cerámica tardoantigua en la cuenca del Duero" en CABALLERO, L.; MATEOS, P.; RETUERCE, M. (eds.), Cerámicas tardorromanas y altomedievales en la Península Ibérica. Ruptura y continuidad, Mérida, 2003; VIGIL-ESCALERA, A., "Algunas observaciones sobre las cerámicas de época visigoda (ss. V-IX d. C.) de la región de Madrid" en MALPICA CUELLO, A.; CARVAJAL LÓPEZ, J. C. (eds.), Estudios de cerámica tardorromana y altomedieval, Granada, 2007, pp. 357-382.

Para el caso salmantino, ver DAHÍ, S., "Un contexto cerámico de la Antigüedad tardía: el yacimiento de San Pelayo (Aldealengua, Salamanca). Nuevos datos sobre la cronología de las pizarras visigodas", Pyrenae, 38,1, 2007, pp. 57-77; ARIÑO, E.; DAHÍ, S.: "Contextos cerámicos de la Antigüedad Tardía y la Alta Edad Media en la provincia de Salamanca (España)", SFECAG, Actes du Congrès de l'Escala-Empúries, 2008, pp. 265-276; ARIÑO, E.; BARBERO, L.; SUÁREZ, M., "Primeros datos sobre análisis arqueométricos de la cerámica de cocina del periodo romano tardío/visigodo de la provincia de Salamanca (España)" en 
GURT, J. Ma' BUXEDA, J.; CAU, M. A. (ed.). Late Roman Coarse Wares, Cooking wares and anphorae in the Mediterranean. Archaeology and Archaeometry. Oxford, 2005; GÓMEZ GANDULLO, J., "Avance sobre las excavaciones arqueológicas en el yacimiento de época visigoda de La Legoriza, San Martín del Castañar (Salamanca)", Zona arqueológica, 8 (1), 2006, pp. 216 235.

${ }^{33}$ Véase la transcripción en HERNÁNDEZ GUERRA, L., Epigrafía de época romana de la provincia de Salamanca, Valladolid, 2001, p. 104.

${ }^{34}$ GARCÍA MARTÍN, J., "La fíbula zoomorfa de caballito hallada en Garcihernández”, Salamanca, Revista de Estudios, 1, 1982, pp. 215-216.

${ }^{35}$ SANTONJA, M.; MORENO, M. y VELÁZQUEZ, I., "Tres pizarras con dibujos de época visigoda en la provincia de Salamanca”, Zephyrus, XLIV-XLV 1991-1992, pp. 471-496.

${ }^{36}$ BENET, N., “Investigaciones Arqueológicas en Castilla y León”, Numantia III, 1990, pp. 285-286.

${ }^{37}$ Un ejemplo de esta metodología, basado en la utilización de tumbas excavadas en roca como marcadores de espacios en el paisaje, puede verse en MARTÍN VISO, I., "Tumbas y sociedades locales en el centro de la Península Ibérica en la Alta Edad Media: el caso de la comarca de Riba Côa (Portugal)", Arqueología y territorio medieval, 14, 2007, pp. 21-48.

${ }^{38}$ DELOGU, P., “Archeologia Medievale...”, p. 505; MORELAND, J., op. cit. 